\title{
Online Fake Review Identification based on Decision Rules
}

\author{
Sanjay K S $\mathbf{1}^{\mathbf{1}}$, Ajit Danti ${ }^{2}$
}

${ }^{1}$ The Bangalore social \& Educational Institutions, India, sanjukumsi@ gmail.com

${ }^{2}$ Christ(Deemed to be University),Bangalore,Karnataka, India, ajit.danti @ christuniversity.in

\begin{abstract}
Posting of online reviews play a dominant role in sharing the customer's opinion in social Medias. But the challenge is how to trust these reviews. Many researchers carried their work on sentimental analysis, predictions or forecasting but very few focused on fake reviews analysis. Fake reviews also change the mood of the people on their buying pattern. In the online shopping at a greater extent. In this paper, several conditions are applied on the reviews to identify fake reviews using support vector machines. Experimental results are validated using various accuracy measures and compared to state of the art methods to demonstrate the efficacy of the proposed method.
\end{abstract}

Key words: fake reviews, online reviews, SVM.

\section{INTRODUCTION}

Today online shopping has changed the way of peoples shopping pattern and share their shopping experiences or views in the social media. Due to which demand for online shopping portals has been increasing day by day due to its availability and convenience. Customer's opinions or reviews influence other customer's lot in their shopping taste and buying decision. Opinion mining helps vendors to increase their sales and attract more customers.

In order to boost up sales, companies post modified reviews for their benefits which is unethical, these attired reviews or fake reviews misguide the customers. Due to growing unhealthy competitions in the industry, there is an acute need of automatic detection of fake reviews to uphold the dignity or good culture in the market.

In this paper, an automatic detection of fake reviews has been proposed based on certain conditions using SVM.

\section{LITERATURE SURVEY}

Researchers proposed several methods in the area of data mining, sentimental analysis, fake review analysis etc. To classify movie reviews into groups of positive or negative polarity by using machine learning algorithms and analysis of online movie reviews using SA methods in order to detect fake reviews has been proposed [2].
A feature set, capturing the user social interaction behavior to identify fraud, the problem being solved is one of the characteristics that lead to fraud rather than detecting fraud has been proposed [4]. Systematically analyzing and categorizing models that detect review spam has been proposed [1]. A simple statistical method to detect online reviews manipulation, and assess how consumers respond to products with manipulated reviews has been proposed by [7]. A supervised machine learning based solution is proposed for an effective spammer detection has been proposed [10] spam detection using personal characteristics rather than the reviews has been proposed [8]. An analysis that emulates an actual attack on a real review corpus has been proposed [9]. A fast and effective method CATCHSYNC, which exploits two of the tell-tale signs left in graphs by fraudsters such as synchronized behavior, has been proposed [3]. A collective classification algorithm called Multi- typed Heterogeneous Collective Classification (MHCC) and then extend it to Collective Positive and Unlabeled learning (CPU) has been proposed [5]. scoring methods to measure the degree of spam for each reviewer and apply them on an Amazon review dataset has been proposed [6].

\section{PROPOSED METHODOLOGY}

Online shopping is in popular now a days, the branded products are affected in market due to fake reviews. The biggest Challenges is to identify the fake reviews. To identify the fake reviews, five different rules are formed based on star ratings, emotional words, Maximum words, Minimum words, product name specified more than once. In this paper online reviews of the product are extracted by using Web harvy crawler. The features are extracted from the reviews further, rules are defined to identify the fake reviews by using SVM classifier. The overall architecture of the proposed Fake review Identification model is as shown below in Figure 1. 


Reviews $\rightarrow \begin{gathered}\text { Feature } \\ \text { Extraction }\end{gathered} \longrightarrow \begin{gathered}\text { Defining } \\ \text { Rules }\end{gathered} \rightarrow \begin{gathered}\text { Apply SVM } \\ \text { Classifier }\end{gathered} \longrightarrow \begin{gathered}\text { Decision } \\ \text { Fake/Not }\end{gathered}$

Figure 1: Block Diagram of the proposed method

\subsection{Defining Rules}

Following rules are defined to identify whether reviews are fake or not.

R1(Only Star but No review): It is to check whether the reviewer has written any reviews or not. Generally if reviewer wants to make a fake review on any particular product some times reviewer will give the star ratings without any review.

R2(Emotional words): This is to check whether the reviewer has used any Emotional words. If the reviewer has used Max Emotional words then it indicates that the review is fake.

- R3(Review contain more than 500 words):This rule is to check whether the review contain more than 500 words. Usually the reviewer will be writing the reviews without any strong words, simply the review contents will be more.

R4(Review contain less than 10 words): This is to check whether the review contain less than 10 words. Usually the reviewer will be writing the Negative/Positive reviews very short without specifying the reason.

R5(Product Name specified more than once): This rules is to check whether the reviewer has used the product name more than once, because the reviewer will highlight the product name frequently in the review to promote the product.

- Figure 2 shows the plot of Support vector machine, under a supervised machine learning Technique which is used for classification. Here the training dataset are linearly separable, by two parallel hyper planes that separates fake and not fake classes. The region between the two hyper planes is called as "margin". The max -margin hyper plane which lies halfway between them, used to classify the reviews into fake and not-fake.

SVM classifier is shown in the Figure 2 for each rule applied in the reviews.

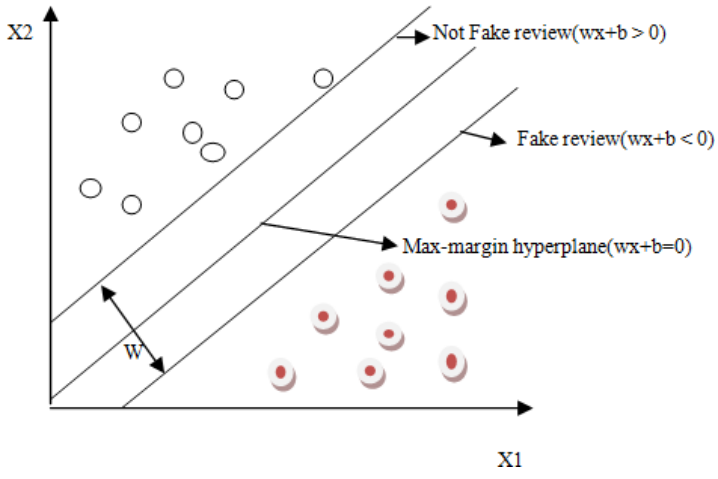

Figure 2: Classification of fake reviews using SVM.

Max margin hyper plane is given by equation (1).

$$
g(x)=\vec{w} \vec{x}+b
$$

Where $\vec{x}$ : Feature vector for particular rule.

$\vec{w}$ : Weight Vector.

$\mathrm{b}:$ constant

$\mathrm{g}(\mathrm{x})$ : hyper plane value.

Any review lies above the maximum margin hyper plane is classified as not fake using the equation (2).

if $g(x)=\left\{\begin{array}{l}>0 \text { review is not fake } \\ <0 \text { review is fake }\end{array}\right.$

Any review lies below the max margin hyper plane is classified as fake review as given in equation (2).

The distance between the two hyper planes will be written as

$$
\frac{2}{\|\vec{w}\|}
$$

To maximize the distance between the two planes $\vec{w}$ is used.

\section{EXPERIMENTAL RESULTS}

The empirical outcome display the performance of the proposed task which covers data acquisition and applying machine learning technique using Support vector machine and to compare the result with the decision tree classifier technique result to identify the best method among these two. Here WEKA tool is used for examination. Thedossierset is braced by assortment of the reviews on the products called "Haier- Refrigerator" from the network www.Amazon.com . 500 online reviews has been collected 
Sanjay K S et al., International Journal of Advanced Trends in Computer Science and Engineering, 8(2), March - April 2019 , 140 - 143

for experimental result, out of which 152 reviews has been detected as fake review by the recommended approach. The recommended approach is validated by manual review analysis, in which 161 reviews are detected as fake by manually. Hence the success rate of $94 \%$ has been achieved as shown in the Table 1.

\begin{tabular}{|c|c|}
\hline \multicolumn{2}{|c|}{ Table 1: Success rate of the proposed method. } \\
\hline Number of Reviews collected & 500 \\
\hline Product & Haier- Refrigerator \\
\hline Website & Amazon \\
\hline $\begin{array}{c}\text { Fake Reviews Identified } \\
\text { Using proposed method } \\
\text { Make Reviews Identified } \\
\text { Manually }\end{array}$ & 152 \\
\hline \begin{tabular}{c} 
Success rate \\
\hline
\end{tabular}
\end{tabular}

The Empirical outcome displays the capability of the recommended system. The Table 2 displays the sample fake reviews of the product.

\begin{tabular}{|c|c|c|c|}
\hline SL.No & Details & Review 1 & Review 2 \\
\hline 1 & PRODUCT & $\begin{array}{c}\text { Haier- } \\
\text { Refrigerator }\end{array}$ & $\begin{array}{c}\text { Haier- } \\
\text { Refrigerator }\end{array}$ \\
\hline 2 & $\begin{array}{c}\text { Only Star but No } \\
\text { review }\end{array}$ & YES & $\mathrm{NO}$ \\
\hline 3 & $\begin{array}{l}\text { emotionalwords } \\
>8\end{array}$ & $\mathrm{NO}$ & YES \\
\hline 4 & $\begin{array}{l}\text { review is more } \\
\text { than } 500 \text { words }\end{array}$ & $\mathrm{NO}$ & YES \\
\hline 5 & $\begin{array}{l}\text { Review Less } \\
\text { than } 10 \text { words }\end{array}$ & YES & NO \\
\hline 6 & $\begin{array}{l}\text { product name } \\
\text { specified more } \\
\text { than once }\end{array}$ & $\mathrm{NO}$ & YES \\
\hline
\end{tabular}

The introduced approach is compared with the other state of art approaches and introduced approach exhibited 94\% success rate as shown in the Table 3.

\begin{tabular}{|l|c|c|c|}
\hline \multicolumn{3}{|c|}{ Table 3: Comparision of proposed method with state of the art. } \\
\hline $\begin{array}{c}\text { Sl } \\
\text { No }\end{array}$ & Authors & $\begin{array}{c}\text { Success } \\
\text { rate }\end{array}$ & $\begin{array}{c}\text { Method } \\
\text { Used }\end{array}$ \\
\hline 1 & $\begin{array}{c}\text { Xianghan } \\
\text { Zheng et.al, 2015 }\end{array}$ & $99.1 \%$ & $\begin{array}{c}\text { Support } \\
\text { Vector } \\
\text { Machine(SVM). }\end{array}$ \\
\hline 2 & $\begin{array}{c}\text { Simran Bajaj } \\
\text { et.al, 2017 }\end{array}$ & $65 \%$ & Novel User-based \\
\hline 3 & $\begin{array}{c}\text { Kunal } \\
\text { Goswami et.al, } \\
2017\end{array}$ & $95 \%$ & $\begin{array}{c}\text { Neural } \\
\text { network } \\
\text { algorithm }\end{array}$ \\
\hline
\end{tabular}

\begin{tabular}{|l|c|c|c|}
\hline 4 & $\begin{array}{c}\text { Esha Tyagi } \\
\text { and Arvind } \\
\text { Kumar Sharma, } \\
2017\end{array}$ & $89.98 \%$ & $\begin{array}{c}\text { Support } \\
\text { Vector } \\
\text { Machine(SVM) }\end{array}$ \\
\hline 5 & $\begin{array}{c}\text { Michael } \\
\text { Crawford, 2015 }\end{array}$ & $98 \%$ & $\begin{array}{c}10 \text {-fold } \\
\text { cross validation }\end{array}$ \\
\hline 6 & $\begin{array}{c}\text { Proposed } \\
\text { method }\end{array}$ & $94 \%$ & $\begin{array}{c}\text { Support } \\
\text { Vector } \\
\text { Machine(SVM). }\end{array}$ \\
\hline
\end{tabular}

\subsection{Evaluation of the proposed method.}

Proposed nearing is gauged by different measures and its certainty is exhibited in the Table 4.

\begin{tabular}{|c|c|c|}
\hline \multicolumn{3}{|c|}{$\begin{array}{l}\text { Table 4: Different Accuracy Measures for the proposed } \\
\text { method. }\end{array}$} \\
\hline Sl.No & Measures & Accuracy in (\%) \\
\hline 1. & Kappa Statistic & $77.55 \%$ \\
\hline 2. & Mean Absolute Error & $12 \%$ \\
\hline 3. & Root Mean Squared Error & $31.72 \%$ \\
\hline
\end{tabular}

Kappa Statistic measures the certainty of the structure using randomness of the data using equation (4).

$$
\text { (Kappa) } K=\frac{a_{0}-a_{e}}{1-a_{e}}
$$

Where 'ao' is monitored certainty and ' $\mathrm{ae}$ ' is expected certainty.

Fleiss contemplates kappa $(\mathrm{K})>0.75$ as exemplary, 0.400.75 as decent to favorable, and $<0.40$ as indigent. here the kappa is 0.77, shows the proposed nearing is exemplary.

Means Absolute Error (MAE) is the moderate outright disparities betwixt classifier anticipated result and substantive result as addicted in the equation (5). Lesser MAE value indicate higher accuracy of the proposed method.

$$
\operatorname{MAE}=\frac{1}{N} \sum_{i=1}^{N}\left(\text { Desired }_{\mathrm{i}}-\text { Actual }_{\mathrm{i}}\right) \leq \epsilon
$$

Similarly Root Mean Square Error (RMSE) is often worn quota of the differences betwixt value anticipated by a exemplary and the values actually examined. RMSD is a acceptable quota of certainty as given by the equation. 


$$
\operatorname{RMSE}=\sqrt{\frac{1}{N} \sum_{i=1}^{N}\left(\text { Desired }_{\mathrm{i}}-\text { Actual }_{\mathrm{i}}\right)^{2}} \leq \epsilon
$$

\section{CONCLUSION}

In this paper, identification of fake reviews on the web products is proposed based on conditions applied on online reviews using SVM Classifier. Results are validated using various accuracy measures such as kappa statistics, Mean Absolute Error (MAE) and Root mean Square Error (RMSE) to determine the robustness of the proposed method. Experimental Result shows that proposed method shows $94 \%$ success against the manual identification of the fake reviews. Proposed approach is also compared to state contemporary method to reveal the virtue of the way.

\section{REFERENCES}

1. Atefeh Heydari, Mohammed ali Tavakoli ,Naomie salim, Zahra Heydari "Detection of review spam: A survey",Expert Systems with Applications, Volume 42, Issue 7, 1 May 2015, Pages 3634- 3642,Elsevier Journal, https://doi.org/10.1016/j.eswa.2014.12.029.

2. Elshrif Elmurngi and Abdelouahed Gherbi "An empirical study on detecting fake reviews using machine learning techniques" Seventh International Conference on Innovative Computing Technology, August 2017 IEEE conference, Pages 107-114,ISBN: 978-1-5090-3990-6.

3. Jiang M, Cui P, Beutel A, Faloutsos C, Yang S. "Catchsync: catching synchronized behavior in large directed graphs" In:Proceedings of the 20th ACM SIGKDD international conference on knowledge discovery and data mining, KDD '14. New York: ACM; 2014. p. 941-50. https://doi.org/10.1145/2623330.2623632

4. Kunal Goswami, Younghee Park ,Chungsik Song "Impact of reviewer social interaction on online consumer review fraud detection" Big Data (2017) 4:15, Springer journal, DOI 10.1186/s40537-017-00756.

5. Li H, Chen Z, Liu B, Wei X, Shao J. "Spotting fake reviews via collective positive-unlabeled learning." Proceedings of the 201 IEEE international conference on data mining, IEEE computer society. Washington: ICDM '14; 2014. p.899-904. https://doi.org/10.1109/ICDM.2014.47

6. Lim EP, Nguyen VA, Jindal N, Liu B, Lauw HW "Detecting product review spammers using rating behaviors" In: Proceedings of the $19^{\text {th }}$
ACM international conference on information and knowledge management. New York: ACM, CIKM '10; 2010. p. 939-48.

7. NanHu, Indranil Bose, Noi SianKoh,LingLiu "Manipulation of online reviews: An analysis of ratings, readability, and sentiments", Decision Support Systems, Volume 52, Issue 3, February 2012, Pages 674-684, Elsevier Journal, https://doi.org/10.1016/j.dss.2011.11.002.

8. Simran Bajaj, Niharika Garg, Sandeep Kumar Singh "A Novel User-based Spam Review Detection", Information Technology and Quantitative Management (ITQM2017), 10.1016/j.procs.2017.11.467, Elsevier Journal. https://doi.org/10.1016/j.procs.2017.11.467

9. Theodoros Lappas "Fake Reviews: The Malicious Perspective", Springer-Verlag Berlin Heidelberg 2012, NLDB 2012, LNCS 7337, pp. 23-34, 2012. https://doi.org/10.1007/978-3-642-31178-9_3

10. Xianghan Zheng , ZhipengZeng, ZheyiChen, YuanlongYu, Chunming Rong, "Detecting spammers on social networks", Neurocomputing,www.elsevier.com/locate/neucom 0925-2312 \& 2015. http://dx.doi.org/10.1016/j.neucom.2015.02.0 47. 